\title{
Predicting maintainability of object-oriented software using metric threshold
}

\begin{abstract}
This study presents the empirical investigation into maintainability of software using Chidamber and Kemmerer Metric suite. The study used metric threshold to propose the model for predicting maintainability of object-oriented software. Two geospatial software systems were used to identify the extent on which the metrics in Chidamber and Kemmerer suite can be used to track the needed efforts during maintenance phase. Linear Discriminant Analysis was used to find the performance measurement for each metric in order to understand their overall effect on particular software product. The results indicated the significant impact of the Chidamber and Kemmerer metrics in predicting maintainability when threshold are used over expertsôopinions. Moreover, the results found the important role of size and inheritance metrics in predicting maintainability of Object-oriented software and highlighted the needs for further empirical investigation. This is specifically on the production of more metrics thresholds that give researchers and practitioners a room to work on more metrics.
\end{abstract}

Keyword: Maintainability; Metric threshold; Expertôs opinions; Object-oriented software; Linear discriminant analysis 